International Journal of Biology, Pharmacy and Allied Sciences (IJBPAS) 'A Bridge Betusen Caboratory and QRader'

WwW.iibpas.com

OXIDATIVE STRESS: A CAUSE OF INFERTILITY IN WOMEN

GUPTA DV ${ }^{1}$, KHURANA HK ${ }^{2}$, BALA $K^{3}$ AND SHUKLA $A^{3 *}$

1: Department of Bioprocess Technology, Institute of Chemical Technology, Mumbai, Maharashtra-400019

2: School of Interdisciplinary Sciences and Technology, Jamia Hamdard University,

New Delhi-110062

3: Amity Institute of Biotechnology, Amity University Uttar Pradesh, Noida, (U.P.) India, 201301

*Corresponding Author: E Mail: ashukla5@amity.edu

Received $19^{\text {th }}$ Nov. 2019; Revised $14^{\text {th }}$ Dec. 2019; Accepted $19^{\text {th }}$ Jan. 2020; Available online $1^{\text {st }}$ July 2020

https://doi.org/10.31032/IJBPAS/2020/9.7.5186

ABSTRACT

There are various reasons that have been identified for the infertility in females and one of them is oxidative stress. Oxidative stress (OS) results when the body is not able to neutralize the generation of free radicals or reactive oxygen species (ROS) like hydroxyl radicals and thus an imbalance is generated between pro-oxidants and anti-oxidants. In the ovary, there is a complex relation between ROS and antioxidants. The environment required in vivo for fertilization and embryo development is of low oxygen tension. A fine balance between pro oxidants and anti-oxidants is required for smooth functioning of female reproductive tract. There are various factors which lead to production of oxidative stress in turn causing infertility. The increased oxidative stress can be detected using various biomarkers which are specific to female reproductive tract. The supplementation of antioxidants can help curb the problem of infertility. This study is focused on the causes, testing and treatment of oxidative stress in females which causes infertility.

Keywords: Oxidative stress, infertility, antioxidants, ROS

\title{
INTRODUCTION
}

Infertility is a condition which can be stated as the inability to conceive and is a significant health concern. The pervasiveness of female infertility is in the range of $7 \%-28 \%$ depending on the age of the woman as reported in Advance Access 
publication June 4, 2008. There can be many reasons of infertility like problems with ovulation leading to hormonal disorders, polycystic ovarian syndrome; problems with fallopian tubes causing pelvic inflammatory disease and oxidative stress [1]. According to a study reported by Agarwal et al, oxidative stress has a vital role in tubal factor, endometriosis, peritoneal factor and unexplained infertility. The generation of free radicals in the body causes oxidative stress [2]. Free radicals have one or more unpaired electrons in their outer shell [3]. Being a highly reactive and unstable species, it acquires an electron from carbohydrates, lipids, nucleic acids, proteins, or any other molecule to become stable. This causes the passing of successive chain reactions which further damages cell causing disease [2].

\section{Causes of oxidative stress}

Oxidative stress (OS) is generated when there is an imbalance between antioxidants and pro-oxidants produced from aerobic metabolism [4]. The Reactive Oxygen Species are the highly reactive species comprising of oxygen radicals such as the hydrogen peroxide $\left(\mathrm{H}_{2} \mathrm{O}_{2}\right)$, superoxide anion $\left(\mathrm{O}^{2-}\right)$, the hydroxyl radical $\left(\mathrm{OH}^{-}\right)$, hypochlorite radical $\left(\mathrm{OHCl}^{-}\right)$and the peroxyl radical $\left(\mathrm{ROO}^{-}\right)$[3]. Overall ROS level is regulated by counteracting with the antioxidant defense system involving catalase (CAT), superoxide dismutase (SOD), and reduced glutathione (GSH) and thus maintaining the physiological homeostasis. But this mechanism gets interrupted during cellular proliferation if the level of ROS reaches below the homoeostatic set point. Also, an elevation in the levels of ROS generates a stress signal which in turn activates the specific redoxsensitive signaling pathways [5]. Antioxidants balance the level of these reactive species in the system and reduce its harmful effects. They stabilise the free radical species by donating an electron without destabilizing itself. The disruption in the balance between anti and pro oxidants, due to either the incapability of the antioxidants to neutralize ROS or over production of ROS, results in oxidative stress as shown in Figure 1 [6].

\section{Role of oxidative stress in causing female infertility}

In living cells amid typical physiological condition, ROS are continuously formed as a result of external factors as well as biochemical reactions [6]. ROS production in the mammalian cells is considered to be necessary due to its contribution in the regressive redox signaling from the organelle to the nucleus and the cytosol [7]. In response to physiological stimuli, there is production of ROS in a limited amount [6]. ROS act as secondary messengers which regulates apoptosis, activates 
transcription factors and modulates the expression of various genes involved in immune response [8]. OS occurs when the generation of ROS and other radical species surpasses scavenging by antioxidants. In the body, OS induces peroxidation of lipids, structurally and functionally modifies protein and DNA, promotes cell death and contributes to the risk of cancer and other chronic diseases [9]. Throughout the female reproductive system, there is a fine balance which exists between antioxidants and ROS. The increase in ROS in the female reproductive system can lead to several gynecological diseases including infertility [6]. One of the reasons of infertility is the over production of ROS in the peritoneal cavity [5]. The sensitivity of gametes towards the damage by ROS is more [9]. The increased ROS, free radials and lipid peroxides causes oxidative stress which in turn has an impact on the fertilisation rate, oocyte penetration, viability of cells and implantation. All this leads to infertility as shown in Figure 2. (a)

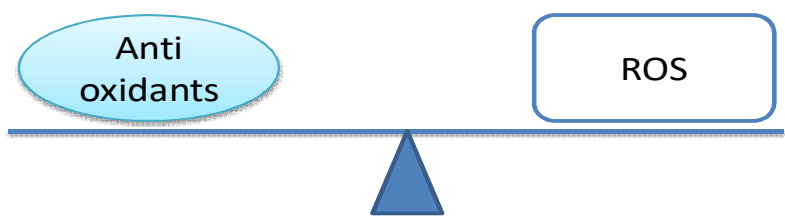

(b)

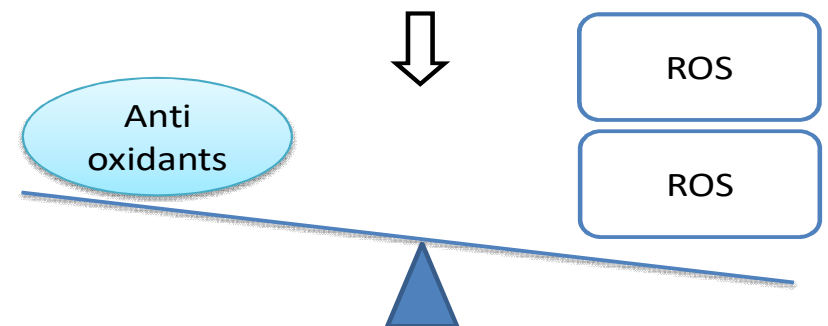

(c)

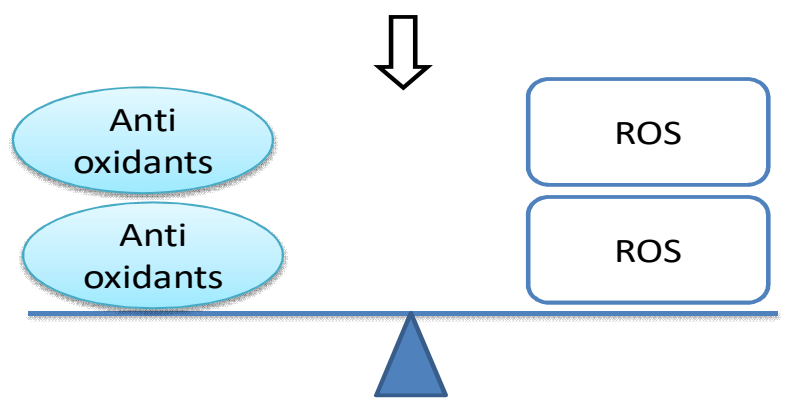

(a) Homeostasis condition is maintained by the balance between anti-oxidants and ROS

(b) Imbalance created between anti-oxidants and ROS which causes oxidative stress.

(c) Balance is created by increase in antioxidants (no oxidative stress)

Figure 1: Relation between reactive oxygen species (ROS), antioxidants and oxidative stress [6] 


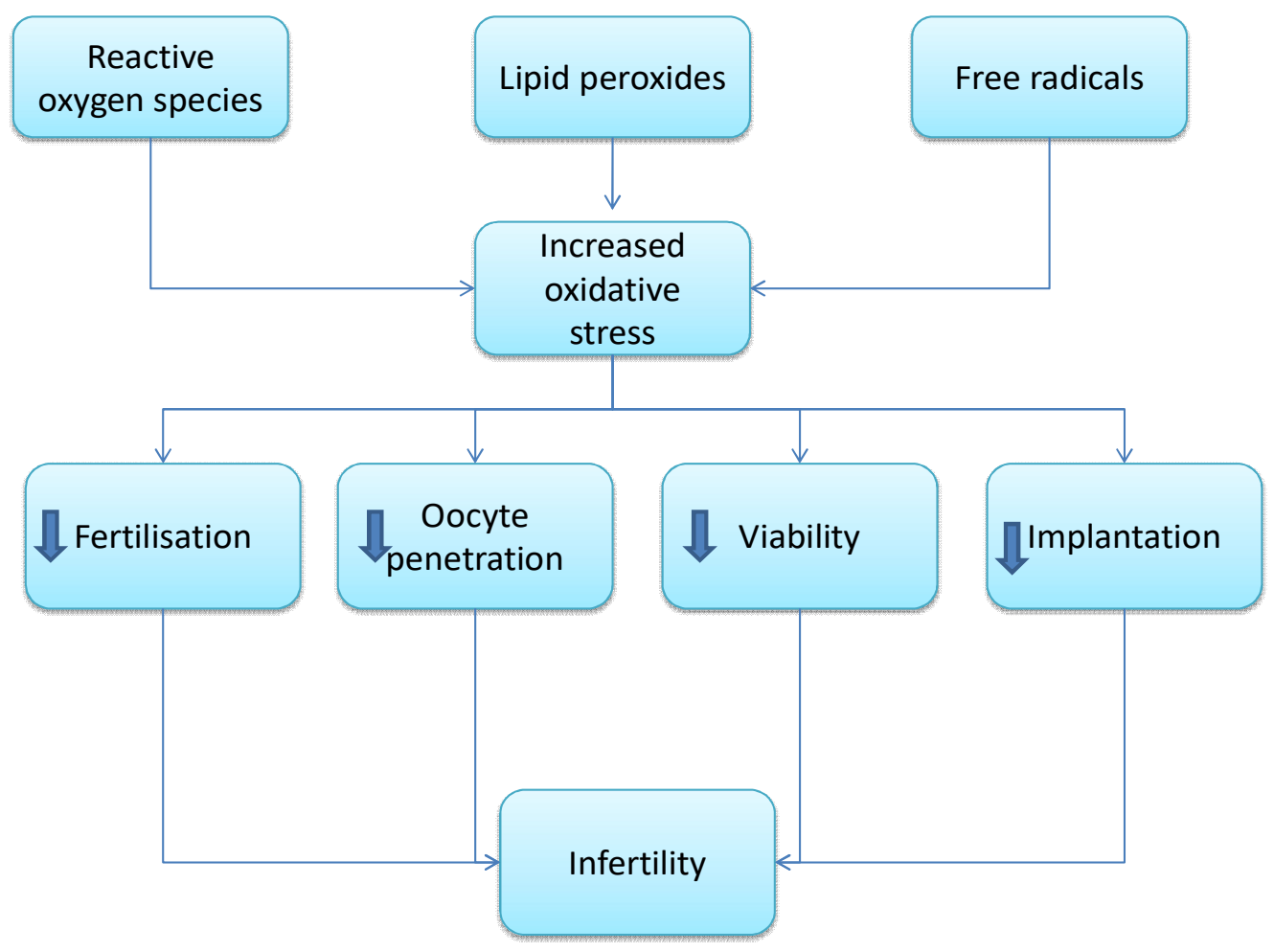

Figure 2 : Effect of oxidative stress in causing female infertility

OS imbalances the levels of progesterone and estrogen which is important for development and maintenance of female reproductive system [10]. Less amount of antioxidant in the system can cause increased chances of abortion [5]. An increased level of ROS has been found having a devastating effect on physiology and pathology of the female reproductive tract. Also, various studies ensure that ROS is present in the female reproductive system [11].

It alters the physiological reproductive functions involving maturation of ooycte, steroidogenesis of the ovary, the function of corpus luteum and luteolysis [12]. These all disorders which affect female fertility have common etiopathogenic mechanisms which initiate from embryo metabolism and its surroundings [2]. Previous findings also suggest that premature rupture of the fetal membranes is due to ROS that means oxidative stress is involved in preeclampsia too [5].

A cohort of oocytes initiates its growth within the ovary each month. However, meiosis $I$ is resumed in only one of them i.e. the dominant oocyte after the increase 
in ROS. Antioxidants interfere with this process. In contrast, antioxidants promote the development of meiosis II. The amount of pro oxidants and antioxidants in the ovary fluctuates and thus they share a complicated relationship. Growing follicle causes production of the steroids leading to an increase in levels of P450. The ROS is produced by the pre-ovulatory follicle which evokes ovulation. The ovarian follicle needs follicular angiogenesis for its appropriate development and growth, stimulated by oxygen deprivation. Apoptosis is promoted by the follicular ROS whereas this action is counterbalanced by follicular stimulating hormone (FSH) and glutathione (GSH) in the growing follicle itself. Ovulation, a necessary process for reproduction, is initiated by the surge in levels of Luteinizing Hormone (LH), which further stimulates some significant physiological changes and an egg is released. Post-LH surge inflammatory precursors play a crucial role in ovulation; its overabundance generates ROS while its depletion impairs ovulation [13]. For a successful pregnancy, progesterone production is indispensable. Progesterone is released by the corpus luteum produced after ovulation in the ovaries. Corpus luteum persists when the pregnancy takes place [14]. If there is no pregnancy, then regression takes place which results in the production of ROS.
Proper development of the follicle in the next cycle requires a rapid decline in the levels of progesterone. Throughout the early to mid-luteal phase, the levels of $\mathrm{Cu}$, $\mathrm{Zn}-\mathrm{SOD}$ in the corpus luteum increases and then lowers in the regression phase. During the regression phase, the levels of progesterone decrease whereas lipid peroxide levels increase. The increase in ROS concentration during regression is because of the reduction in the concentration of $\mathrm{Cu}, \mathrm{Zn}-\mathrm{SOD}$. The concentration of $\mathrm{Cu}, \mathrm{Zn}-\mathrm{SOD}$ gets decreased due to an increase in theconcentration of macrophages; that revive the generation of the superoxide (SO) anion by luteal cells and phagocytic leukocytes within the corpus luteum or, a decrease in blood flow of ovaries; which produces ROS and damages tissues. During regression, the concentration of Mn-SOD within the corpus luteum gets increased. This Mn-SOD with the help of inflammatory reactions and cytokines scavenges the ROS produced in the mitochondria. Due to this, the concentration of Mn-SOD gets reduced in the regressed cell. Thereupon, apoptosis is forthcoming. The $\mathrm{Cu}, \mathrm{Zn}-\mathrm{SOD}$ enzyme is closely associated with progesterone generation, whereas Mn-SOD provides protection to luteal cells from OS-induced inflammation. ROS levels increase due to the metabolism of an oocyte and an 
insufficient amount of antioxidants within the oviductal and follicular fluid of the embryo. The condition of oocytes and their communication with sperms are affected by the oocyte development in the follicular fluid environment where ROS levels are high. This affects implantation and embryo development [13].

The ovary is a metabolically active organ and thus, is repeatedly under stressed conditions. During ovulation, ROS has a physiological role which is identical to inflammation. Ovulation is kept in check by agents that prevent acute inflammatory reactions from occurring. The involvement of ROS within this process is proved as it is released in connection with follicle rupture. The macrophages and neutrophils are the inflammatory cells present in the ovary at ovulation. They are the source of ROS and produce a large number of free radicals. The corpus luteum produces abundant progesterone collectively with the reaction of P450s by consumption of molecular oxygen and thus, eventually produces ROS as a byproduct. In the ovary, ovulation is hampered by superoxide dismutase (SOD) which is produced to repress ROS. The levels of ROS in the corpus luteum increase during the regression phase. The NADPHdependent generation of superoxide in the ovary also increases during the early preovulatory phase in cycling females. Therefore, this concludes that oxidative stress causes problems with female fertility [12].

\section{Effect of oxidative stress on embryo}

It is reported that several teratogens influence the developing fetus by increasing the oxidative stress which ends up in extreme damage to the embryo. This mechanism works in diabetic-induced embryonic damage, cocaine, ionizing radiation causing teratogenicity, cigarette smoking, hypoxia and alcohol. Clinical studies demonstrate that the fundamental process of fetal damage, prompted by elevated amounts of previously mentioned factors, is also increased by the embryonic OS. In the same way, teratogenic activity of many drugs is through embryonic OS [15]. Abnormal placentation may also cause enhanced placental OS, resulting in embryonic death. Animal studies demonstrate that administration of various antioxidants is effective in lowering the damaging impact of heightened OS actuated by teratogens [16].

\section{MATERIALS AND METHODS}

\section{Tests to determine oxidative stress}

\section{in females}

Peritoneal fluids, follicular fluid and amniotic fluid are the various fluids in which most of the biomarkers are observed as well as examined. The ROS can be measured in all these fluids by various tests including nitrobluetetrazolium staining, epifluorescence microscopy, flow 
cytometry, measurement of oxidative DNA adducts, Enzyme-Linked Immunosorbent Assay (ELISA),Near-Infrared Spectroscopy and Raman analysis [17].

\section{Biomarkers of oxidative stress}

ROS is considered as the initial marker and to measure the damage induced such as lipid peroxidation, DNA damage and oxidation of proteins, some other markers are available. For the proper investigation of the role of ROS and oxidative stress in the pathogenesis and progression of human diseases, use of appropriate biomarkers is necessary as shown in Table 1. The nature of ROS plays a notable role in determining the use of biomolecules like proteins, lipids, or DNA as a marker of oxidative stress [18].

\section{Biomarkers of OS present in the} reproductive tract of females

There are many biomarkers which have been studied in the reproductive tract of the females, some of which are mentioned in
Table 2 [19]. Various studies confirm presence of ROS and various antioxidant enzymes in the female reproductive tract.

OS disturbs the physiological aspects of ovarian function. According to studies, it has been confirmed that the ROS has a vital role in the functioning of corpus luteum, follicular maturation, ovulation and folliculogenesis. The parenchymal steroidogenic cells, endothelial cells and phagocytic macrophages within the ovaries mark the production of the ROS. However, the biomarkers of normal ovaries such as $\mathrm{Cu}-\mathrm{SOD}, \mathrm{Zn}-\mathrm{SOD}$ and lipid peroxides can be determined by IHC staining and many other methods. It is reported that a decrease in the antioxidant levels, of GPx does not affect the fertilization rates of gametes. Thus, GPx and Mn-SOD can be considered as markers to observe oocyte maturation [17]. The OS biomarkers were investigated along with their measurement units as mentioned in Table 3.

\begin{tabular}{|c|c|}
\hline BIOMOLECULE & MARKER \\
\hline 1. Lipids & $\begin{array}{ccc}- & \text { Chlorinated/nitrated lipids (isoprostanes, isoleukotrienes) } \\
\bullet \quad \text { Oxysterols (aldehyde) } \\
\text { - } & \text { Peroxides (malondialdehyde, 4-hydroxy-2-nominal, acrolein) } \\
\end{array}$ \\
\hline 2. Proteins & $\begin{array}{cc} & \text { Aldehyde adducts } \\
& \text { Carbonyl group formation } \\
\text { - Nitrated/chlorinated Tyr, Trp, Phe }\end{array}$ \\
\hline 3. DNA & $\begin{array}{l}\text { - } \\
\text { - } \\
\text { Nitratehyde/other base adducts } \\
\text { - Oxidized bases }\end{array}$ \\
\hline
\end{tabular}

Table 2: Biomarkers present in the female reproductive tract [20]

\begin{tabular}{|c|c|}
\hline REPRODUCTIVE TRACT SITE & BIOMARKERS \\
\hline$\bullet \quad$ Ovary & $\begin{array}{c}\text { Superoxide Dismutase; CuZn SOD; Mn SOD; Glutathione } \\
\text { peroxidase; lipid peroxidases; }\end{array}$ \\
\hline$\bullet \quad$ Endometrium & Superoxide Dismutase; iNOS; and eNOS. \\
\hline$\quad$ Fallopian tubes & ROS; Superoxide Dismutase; iNOS; and eNOS. \\
\hline$\bullet \quad$ Placenta & Lipid hydroperoxides; Intracellular ROS; \\
\hline$\bullet \quad$ Peritoneal fluid(in endometriosis) & $\begin{array}{c}\text { Oxidized lipoproteins; Intraperitoneal fluid ROS; and Total } \\
\text { Antioxidant Capacity }\end{array}$ \\
\hline
\end{tabular}


Table 3: Oxidative stress biomarkers in the reproductive tract of females [2]

\begin{tabular}{|c|c|c|c|c|}
\hline S. No. & BIOMARKER & METHODOLOGY & $\begin{array}{c}\text { MEASUREMENT } \\
\text { UNITS }\end{array}$ & $\begin{array}{c}\text { REFEREN } \\
\text { CE }\end{array}$ \\
\hline 1) & $\begin{array}{c}\text { Cu SOD, Mn SOD, catalase, } \\
\text { glutathione peroxidase, i.e. } \\
\text { the enzymatic antioxidants }\end{array}$ & $\begin{array}{c}\text { Transcription-polymerase } \\
\text { chain reaction is reversed }\end{array}$ & Sequences of cDNA & $\begin{array}{c}\text { Sugino et. } \\
\text { al [21] }\end{array}$ \\
\hline 2) & Total capacity of antioxidants & $\begin{array}{c}\text { chemiluminescence assay is } \\
\text { enhanced }\end{array}$ & Trolox equivalents & $\begin{array}{c}\text { Attaran et. } \\
\text { al [22] }\end{array}$ \\
\hline 3) & $\begin{array}{c}\text { Peroxides of lipids; } \\
\text { Malondialdehyde, conjugated } \\
\text { dienes, Thiobarbituric acid }\end{array}$ & $\begin{array}{c}\text { Thiobarbituric acid method } \\
\text { Micromole of } \\
\text { malondialdehyde/L }\end{array}$ & $\begin{array}{c}\text { Jozwik et. } \\
\text { al [23] }\end{array}$ \\
\hline 4$)$ & Oxidative DNA adducts & 8-hydroxy 2-deoxyguanosine & $\begin{array}{c}\text { Immunocytochemical } \\
\text { staining }\end{array}$ & $\begin{array}{c}\text { Seino et } \\
\text { al [24] }\end{array}$ \\
\hline
\end{tabular}

\section{Malondialdehyde (MDA) as a}

\section{biomarker}

Lipid peroxidation is oxidative damage of lipids. The generation of lipid peroxides at increased levels causes the production of malondialdehyde (MDA). Nowadays MDA is used for assessment of lipid peroxidation in many expert types of research as an oxidative stress marker. Thiobarbituric Acid Reactive Substance (TBARS) is the oxidative stress marker which binds to MDA. The thiobarbituric acid reactive substance is a very sensitive method for quantitative determination of lipid peroxidation degree, being a key indicator of oxidative stress. The principle of this screening method is that two molecules of thiobarbituricacid reactive substance react with malondialdehyde to create a complex, which is determined by spectrophotometry [25].

\section{Protein carbonyl (CO) group as a}

\section{biomarker}

The consumption of the protein carbonyl groups as oxidative stress biomarkers is meant to be advantageous when compared to the measurement of other oxidation products because of the formation of the relative early protein and also the relative stability of carbonylated proteins. Carbonyl groups are generated by the oxidation of the protein side chain i.e. aldehydes and ketones. These moieties are useful for both their detection and storage because of their chemically stable nature. Majority of protein carbonyl assays do not require any special equipment. The phenomenon of formation of protein-bound $\mathrm{CO}$ groups is more frequent than the lipid peroxidation. Thus using $\mathrm{CO}$ groups as a marker is beneficial [18].

\section{Antioxidant activity naturally}

present in the ovary

\section{Superoxide dismutase (SOD)}

The variable activity of SOD is present in the human pre-ovulatory ovarian follicular fluid and safeguards oocytes against oxidative harm [3]. The contribution of $\mathrm{Cu}$, ZnSOD prevents the aggregation of superoxide radicals which is synthesized by PGF $2 \alpha$ and thus pregnancy is maintained. During pregnancy, the stimulation of luteal 
$\mathrm{Cu}, \mathrm{Zn}-\mathrm{SOD}$ expression by Human

Chorionic Gonadotropin (HCG) could also be vital for sustaining luteal cell integrity [12].

\section{Glutathione (GSH)}

GSH is that biomarker of oxidative stress that detoxifies the produced ROS along with antioxidative enzymes. It scavenges the free radicals present either through reduction of peroxides or direct chemical reactions [27]. The embryo development and quality of oocyte is dependent on GSH which is there in oviductal fluids [28]. The glutathione reductase (GR) is present in the epithelia of the oviducts at high levels. During ovulation, an environment of excess ROS is present and thus to protect oocytes, GSH is secreted. This maintains the efficiency of fertilization. Thus, for a healthy reproductive system, the subsistence of a high redox potential is required [12].

\section{RESULTS}

\section{Drugs used for the treatment}

\section{Melatonin}

Effective improvement of oocyte quality requires treatment of melatonin with a $3 \mathrm{mg}$ tablet which is sufficient to have an increase in the concentrations of intrafollicular melatonin. Melatonin protects the oocytes from ROS. The over production of ROS can damage oocyte quality. Oxidative stress induces caspasemediated cell death of human oocytes [2].
When injected systemically, melatonin enters all the tissues but ovaries actively uptake melatonin and thus there are high levels of it in the preovulatory follicles. Melatonin is a scavenger of free radical. It also regulates gene transcription for antioxidant enzymes. Melatonin may prevent mitochondrial DNA damage in oocytes by improving electron transport chain resulting in improvement of oocyte quality. Melatonin generates various metabolites like cyclic 3hydroxmelatoninand N1-acetyl-5methoxykynuramine (AMK) through different pathways which are equally good scavengers of reactive species. This cascade reaction is the unique property of melatonin because of which it is preferred over other antioxidants. Because of involvement of many metabolites, interaction of the melatonin with ROS becomes prolonged. Thus, use of melatonin is an effective way to protect cells from oxidative stress with minimum side effects [2].

\section{Vitamin E}

Vitamin $\mathrm{E}$ is a fat-soluble vitamin which can protect cell membranes from free radicals. In human, the most active form of Vitamin $\mathrm{E}$ is $\alpha$-tocopherol which is also an effective antioxidant. Lipid hydroperoxides are generated from the phospholipids of cell membrane due to the presence of ROS. Because of generation of these lipid 
molecules, which act as signaling molecules, apoptosis is induced. Vitamin $\mathrm{E}$ has the ability to suppress the production of these lipid molecules. Oxidative damage can be reduced by using Vitamin $\mathrm{E}$ in combination with other antioxidants like melatonin, carotene, Vitamin $\mathrm{C}$, etc. The amount of Vitamin E used is $600 \mathrm{mg}$ /day.

But at DNA level, melatonin has been proved better than Vitamin $\mathrm{E}$ for reduction in molecular damage levels. In conclusion, melatonin protects oocytes effectively from the toxic effects of oxidative stress on oocyte maturation. Thus a combination of melatonin, which improves oocyte quality, and Vitamin E, which works well with other antioxidants, can be administered to infertile women [2].

\section{Vitamin C}

The oxidative stress can be treated in the body by fine tuning the levels of ROS generation and availability of number of antioxidants. Studies reveal that to protect against oxidative stress, the utilization of antioxidants as well as nutritional supplements like vitamin $\mathrm{C}$ has proved beneficial [2]. In case of increased levels of ROS, antioxidants minimize the oxidative damage, repair it or prevent it by neutralizing it [26]. In a recent multi-center study, it was observed that the group of females undergoing treatment of luteal phase defect had higher pregnancy rates due to supplementation of Vitamin $\mathrm{C}$ at 750 $\mathrm{mg}$ /day concentration as compared to that of control.The effect of a nutritional supplement containing selenium, iron, Vitamin E, L-arginine, zinc was tested and the results showed increased levels of midluteal progesterone which increased the ovulation as well as pregnancy rates in the patients. [2].

\section{DISCUSSION}

The oxidative stress plays a fundamental role in causing female infertility. Knowing this fact, the females who are suffering from the problem of infertility, can be tested for the levels of ROS. The tests, which are proposed for detecting increased levels of ROS in the female reproductive tract, are able to do so. The tests which involve the use of biomarkers are specific to the reproductive organs and thus the interference from other organs is eliminated. Due to the threat that oxidative stress poses to the female reproductive tract, it is necessary to curb it. Thus, various vitamins supplements, which have proved to act as antioxidants, have been looked into. To sum up, there is a need of further research for the early detection of the ROS levels and the drugs that can be used to reduce oxidative stress without having any side effects.

\section{Acknowledgements}

We would like to take this opportunity to thank Amity Institute of Biotechnology (Amity University Uttar Pradesh, Noida) 
for providing us the infrastructure to support this study. We especially thank Dr.

Kumud Bala for her valuable suggestions. REFERENCES

[1] Ruder, E. H., Hartman, T. J., Blumberg, J., \& Goldman, M. B. (2008). Oxidative stress and antioxidants: exposure and impact on female fertility. Human reproduction update, 14(4), $345-$ 357.https://doi.org/10.1093/humupd/d mn011

[2] Agarwal, A., Gupta, S., \& Sharma, R. K. (2005). Role of oxidative stress in female reproduction. Reproductive biology and endocrinology, 3(1), 28.10.1186/1477-7827-3-28

[3] Tamura, H., Takasaki, A., Miwa, I., Taniguchi, K., Maekawa, R., Asada, H \& Morioka, H. (2008). Oxidative stress impairs oocyte quality and melatonin protects oocytes from free radical damage and improves fertilization rate. Journal of pineal research, 44(3), 280-287. https://doi.org/10.1111/j.1600079X.2007.00524.X

[4] H Sekhon, L., Gupta, S., Kim, Y., \& Agarwal, A. (2010). Female infertility and antioxidants. Current Women's Health Reviews, 6(2), 8495.https://doi.org/10.2174/157340410 791321381
[5] Rahman, T., Hosen, I., Islam, M. T., \&Shekhar, H. U. (2012). Oxidative stress and human health. Advances in Bioscience and Biotechnology, 3(07), 997.10.4236/abb.2012.327123

[6] Agarwal, A., Gupta, S., \& Sharma, R. (2005). Oxidative stress and its implications in female infertility-a clinician's perspective. Reproductive biomedicine online, 11(5), 641650.https://doi.org/10.1016/S14726483(10)61174-1

[7] Murphy, M. P. (2009). How mitochondria produce reactive oxygen species. Biochemical journal, 417(1), 13.https://doi.org/10.1042/BJ2008138 6

[8] Pincemail, J., Vanbelle, S., Gaspard, U., Collette, G., Haleng, J., CheramyBien, J. P., \& Limet, R. (2007). Effect of different contraceptive methods on the oxidative stress status in women aged 40-48 years from the ELAN study in the province of Liege, Belgium. Human Reproduction, 22 (8), 2335-2343. https: //doi. org/10.1093/humrep/dem146

[9] Mathew, B. B., Tiwari, A., \&Jatawa, S. K. (2011). Free radicals and antioxidants: A review. Journal of Pharmacy Research,4(12), 43404343. 
[10] Shukla, Akshara., Jamwal, Rohitash., \& Bala, Kumud. (2017). Adverse effect of combined oral contraceptive pills. Asian J Pharma \&Clin Res, 10, 1721.https://doi.org/10.22159/ajpcr.20 17.v10i1.14565

[11] Agarwal, A., \& Allamaneni, S. S. (2004). Role of free radicals in female reproductive diseases and assisted reproduction. Reproductive biomedicine online, 9(3), 338 347.https://doi.org/10.1016/S14726483(10)62151-7

[12] Fujii, J., Iuchi, Y., \& Okada, F. (2005). Fundamental roles of reactive oxygen species and protective mechanisms in the female reproductive system. Reproductive biology and endocrinology, 3(1), 43. https://doi.org/10.1186/14777827-3-43

[13] Agarwal, A., Aponte-Mellado, A., Premkumar, B. J., Shaman, A., \& Gupta, S. (2012). The effects of oxidative stress on female reproduction: a review. Reproductive biology and endocrinology, 10(1),

49.https://doi.org/10.1186/14777827-10-49

[14] De Groote, D., d'Hauterive, S. P., Pintiaux, A., Gerday, C., \&Foidart, J. M. (2009). Effects of oral contraception with ethinylestradiol and drospirenone on oxidative stress in women 18-35 years old. Contraception, 80(2), $\quad$ 187193.https://doi.org/10.1016/j.contrac eption.2009.02.015

[15] Ornoy, A. (2007). Embryonic oxidative stress as a mechanism of teratogenesis with special emphasis on diabetic embryopathy. Reproductive toxicology, 24(1), 3141.https://doi.org/10.1016/j.reprotox .2007 .04 .004

[16] Kovacic, P., \& Somanathan, R. (2013). Broad overview of oxidative stress and its complications in human health. Open Journal of Preventive Medicine, 3(01), 32. 10.1128/AAC.00328-11

[17] Sharma, R. K., Reynolds, N., Rakhit, M., \& Agarwal, A. (2013). Methods for detection of ROS in the female reproductive system. In Studies on Women's health (pp. 33-60). Humana Press, Totowa, NJ. https://doi.org/10.1007/978-162703-041-0 2

[18] Dalle-Donne, I., Rossi, R., Giustarini, D., Milzani, A., \& Colombo, R. (2003). Protein carbonyl groups as biomarkers of oxidative stress. Clinica chimica acta, 329(1-2), 23-38. https: //doi. 
org/10.1016/S0009-8981(03)000032

[19] Gupta, S., Malhotra, N., Sharma, D., Chandra, A., \& Ashok, A. (2009). Oxidative stress and its role in female infertility and assisted reproduction: clinical implications.

[20] Agarwal, A. S. H. O. K., \& Gupta, S. A. J. A. L. (2005). Role of reactive oxygen species in female reproduction and the effects of antioxidant supplementation-Part 2. Agro Food Industry $\mathrm{Hi}$ Tech, 16(4), 38.

[21] Sugino, N., Takiguchi, S., Kashida, S., Karube, A., Nakamura, Y., \& Kato, H. (2000). Superoxide dismutase expression in the human corpus luteum during the menstrual cycle and in early pregnancy. Molecular human reproduction, 6(1), 19-25. https: //doi.org/ 10.1210/jcem.85.10.6888

[22] Attaran, M., Pasqualotto, E., Falcone, T., Goldberg, J. M., Miller, K. F., Agarwal, A., \& Sharma, R. K. (2000). The effect of follicular fluid reactive oxygen species on the outcome of in vitro fertilization. International journal of fertility and women's medicine, 45(5), 314-320.

[23] Jozwik, M., Wolczynski, S., Jozwik, M., \& Szamatowicz, M. (1999).
Oxidative stress markers in preovulatory follicular fluid in humans. Molecular

Human Reproduction, 5(5), 409413.https://doi.org/10.1093/molehr/ 5.5.409

[24] Seino, T., Saito, H., Kaneko, T., Takahashi, T., Kawachiya, S., \&Kurachi, H. (2002). Eighthydroxy-2'-deoxyguanosine in granulosa cells is correlated with the quality of oocytes and embryos in an in vitro fertilization-embryo transfer program. Fertility and sterility, 77(6), 1184-1190. https: //doi.org/ 10.1016/S0015-0282(02) 03103-5

[25] Draganovic, D., Lucic, N., \& Jojic, D. (2016). Oxidative stress marker and pregnancy-induced hypertension. Medical Archives, 70 (6), 437.10.5455/ medarh. 2016.70. 437-440

[26] Allamaneni, S. S. (2004). Oxidants and antioxidants in human fertility. Middle East Fertility Society Journal, 9(3).

[27] Devine, P. J., Perreault, S. D., \&Luderer, U. (2012). Roles of reactive oxygen species and antioxidants in ovarian toxicity. Biology of reproduction, $86(2), \quad 27-$ 
1.https://doi.org/10.1095/biolreprod. 111.095224

[28] Agarwal, A., Durairajanayagam, D., \& Du Plessis, S. S. (2014). Utility of antioxidants during assisted reproductive techniques: an evidence based review. Reproductive Biology and Endocrinology, 12(1), 112. https://doi.org/10.1186/1477-7827-12112

[29] Sabatini, L., Wilson, C., Lower, A., Al-Shawaf, T., \& Grudzinskas, J. G. (1999). Superoxide dismutase activity in human follicular fluid after controlled ovarian hyper stimulation in women undergoing in vitro fertilization. Fertility and sterility, 72(6), 1027-1034. https:// doi.org / / $\quad$ /S00150282(99)00411-2

[30] Monaghan, P., Metcalfe, N. B., \& Torres, R. (2009). Oxidative stress as a mediator of life history trade-offs: mechanisms, measurements and interpretation. Ecology letters, 12(1), 75-92. https://doi.org /10.1111/j. 14610248.2008.01258.x 